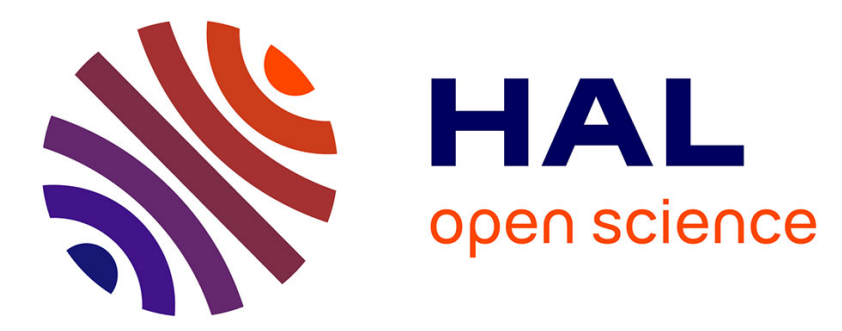

\title{
Multiple modal and wavelength conversion process of a 10-Gbit/s signal in a 6-LP-mode fiber
}

\author{
H. Zhang, M. Bigot-Astruc, Laurent Bigot, P. Sillard, J. Fatome
}

\section{To cite this version:}

H. Zhang, M. Bigot-Astruc, Laurent Bigot, P. Sillard, J. Fatome. Multiple modal and wavelength conversion process of a 10-Gbit/s signal in a 6-LP-mode fiber. Optics Express, 2019, 27 (11), pp.15413. 10.1364/OE.27.015413 . hal-03030011

\section{HAL Id: hal-03030011 https://hal.science/hal-03030011}

Submitted on 10 Dec 2020

HAL is a multi-disciplinary open access archive for the deposit and dissemination of scientific research documents, whether they are published or not. The documents may come from teaching and research institutions in France or abroad, or from public or private research centers.
L'archive ouverte pluridisciplinaire HAL, est destinée au dépôt et à la diffusion de documents scientifiques de niveau recherche, publiés ou non, émanant des établissements d'enseignement et de recherche français ou étrangers, des laboratoires publics ou privés. 


\title{
Multiple modal and wavelength conversion process of a 10-Gbit/s signal in a 6-LP-mode fiber
}

\author{
H. ZHANG ${ }^{1}$ M. BigOt-Astruc, ${ }^{2}$ L. BigOt, ${ }^{3}$ P. SilLLARD, ${ }^{2}$ AND J. \\ FATOME ${ }^{1,{ }^{*}}$ \\ ${ }^{1}$ Laboratoire Interdisciplinaire Carnot de Bourgogne, UMR 6303 CNRS-Université Bourgogne \\ Franche-Comté, 9 av. A. Savary, 21078 Dijon Cedex, France \\ ${ }^{2}$ Prysmian Group, Parc des Industries Artois Flandres, Haisnes 62092, France \\ ${ }^{3}$ Université de Lille, CNRS, UMR 8523, PhLAM-Physique des Lasers Atomes et Molécules, F-59000 \\ Lille, France \\ *Julien.Fatome@u-bourgogne.fr
}

\begin{abstract}
We demonstrate experimentally a simultaneous threefold modal and wavelength conversion process of a $10-\mathrm{Gbit} / \mathrm{s}$ On/Off keying signal in a 1.8 -km long graded-index 6 -LPmode fiber. The principle of operation is based on a phase-matched inter-modal four-wave mixing phenomenon occurring between the fundamental mode and 3 higher-order modes of the fiber. The converted signals show well-opened eye-diagrams and error-free processing.
\end{abstract}

(c) 2019 Optical Society of America under the terms of the OSA Open Access Publishing Agreement

\section{Introduction}

In order to match the continuously increasing data traffic in optical networks and to prevent a "capacity crunch" in the next decades, space-division multiplexing (SDM) has emerged as a breakthrough technology for modern optical communications [1-4]. In this context, multicore fibers (MCF) and few-mode fibers (FMF) or combination of thereof have rapidly emerged as the ideal solution to fulfil the growing demand of capacity [1,2]. Indeed, in such a system, each fiber core or mode represents an independent information channel that operates in parallel with the others, thus enabling to largely overcome the capacity limit imposed on single-mode platforms [5]. In parallel, the development of SDM and associated multimode components and fibers open a new avenue for all-optical processing of spatially multiplexed signals, then pushing the researchers to re-visit and exploit nonlinear effects such as intermodal four-wave mixing (IFWM) [6-11]. The key point is that the different spatial modes are generally characterized by a non-equal propagation constant and thus provides an additional degree of freedom for phase-matching condition compared to the single mode configuration. Hence, efficient four-wave mixing interactions can be involved over a large bandwidth without the restriction to operate near the zero-dispersion wavelength as in parametric processing encountered in single mode highly nonlinear fibers $[12,13]$. The drawbacks, however, are the weak modal overlap coefficients between certain modes, the high level of chromatic and modal dispersions as well as the random coupling between the modes which presently limit the performance of such nonlinear process. Nevertheless, more than 30 years after the pioneer experiments reported by R. H. Stolen and associates [14], the development of SDM technology has generated a renew of interest for IFWM with experimental demonstrations of signal conversion in km-long FMF fibers [9-11].

In this new contribution, we exploit a multiple IFWM phenomenon occurring in a $1.8-\mathrm{km}$ long graded-index 6-LP-mode fiber supporting 4 mode-groups, i.e. $\mathrm{LP}_{01}, \mathrm{LP}_{11 \mathrm{a}}+\mathrm{LP}_{11 \mathrm{~b}}, \mathrm{LP}_{21 \mathrm{a}}$ $+\mathrm{LP}_{21 \mathrm{~b}}+\mathrm{LP}_{02}$ and $\mathrm{LP}_{31 \mathrm{a}}+\mathrm{LP}_{31 \mathrm{~b}}+\mathrm{LP}_{12 \mathrm{a}}+\mathrm{LP}_{12 \mathrm{~b}}$, and report a simultaneous threefold modal and wavelength conversion process of a 10-Gbit/s On/Off Keying signal in the C-band. The conversion process is based on a parallel Bragg-scattering phase-matched IFWM process occurring between the $\mathrm{LP}_{01}, \mathrm{LP}_{11}, \mathrm{LP}_{02}$ and $\mathrm{LP}_{31}$ spatial modes. We have chosen this 
particular configuration of modes to illustrate the proof-of-principle experiment but note that similar results could be achieved with any other set of higher-order modes. The conversion efficiency for each IFWM process has been characterized as a function of the initial signal wavelength and resulting converted signals in higher-order modes show well-opened eyediagrams and error-free processing. Our experimental observations are qualitatively explained by modeling each pair of modes with an independent set of two-mode coupled Nonlinear Schrödinger Equations (NLSE). However, the effective simulation of random polarizationand space- couplings between degenerate modes within the FMF undertest would be needed to get a better description of the underlying multiple IFWM processes.

\section{Theory}

In this section the phase-matching condition for the simultaneous inter-modal four-wave mixing processes occurring in the graded-index 6-LP-mode fiber is first introduced. Then a set of coupled scalar NLSEs is used to give a qualitative analysis of the conversion efficiency of this multiple IFWM process. The impact of fiber birefringence and random coupling between degenerate modes within the same mode-group is also briefly discussed.

\subsection{Phase-matching conditions}
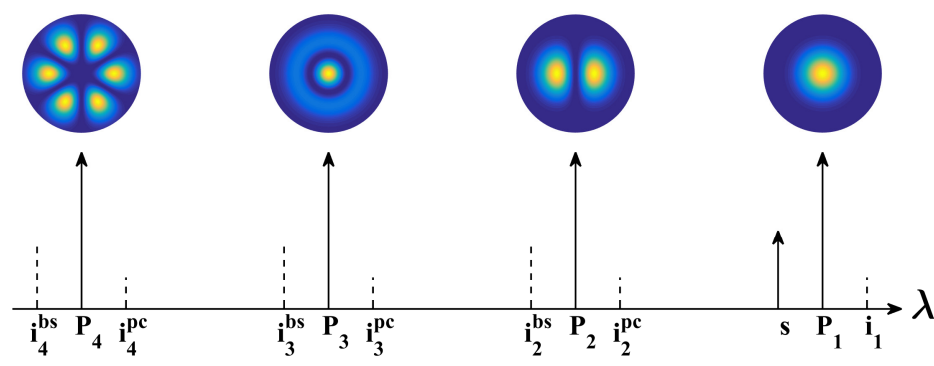

Fig. 1. The input configuration. Four high power $\mathrm{CW}$ pump waves $\mathrm{P}_{1-4}$ are injected into the $\mathrm{LP}_{01}, \mathrm{LP}_{11}, \mathrm{LP}_{02}, \mathrm{LP}_{31}$ modes respectively, together with a weak signal wave injected into the $\mathrm{LP}_{01}$ mode. The generated idler waves are labelled by dashed lines.

The non-degenerate IFWM involving four different wavelengths is investigated in this work. Four high power continuous-waves $\mathrm{P}_{1-4}$ are respectively injected into the $\mathrm{LP}_{01}, \mathrm{LP}_{11}, \mathrm{LP}_{02}$ and $\mathrm{LP}_{31}$ modes of the 6-LP-mode fiber, together with a weak signal wave injected into the $\mathrm{LP}_{01}$ mode (annotated by the solid arrows in Fig. 1). Two important IFWM processes between each higher-order mode and the fundamental mode, namely the Bragg-scattering (BS) and phaseconjugation (PC) are illustrated in Fig. 1. Following the energy conservation law, the corresponding idler frequencies in each higher-order mode are determined as:

$$
\begin{aligned}
& \omega_{\mathrm{i}_{n}}^{\mathrm{bs}}=\omega_{\mathrm{P}_{n}}-\omega_{\mathrm{P}_{1}}+\omega_{\mathrm{s}} \\
& \omega_{\mathrm{i}_{n}}^{\mathrm{pc}}=\omega_{\mathrm{P}_{n}}+\omega_{\mathrm{P}_{1}}-\omega_{\mathrm{s}}
\end{aligned}
$$

where $n=2,3,4$ for the $\mathrm{LP}_{11}, \mathrm{LP}_{02}, \mathrm{LP}_{31}$ modes respectively.

The propagation constant of frequency $\omega$ in the mode $\mathrm{LP}_{i}$ can be expressed as a Taylor series around the common reference frequency $\omega_{0}$ :

$$
\beta^{i}(\omega)=\beta_{0}^{i}+\beta_{1}^{i}\left(\omega-\omega_{0}\right)+\frac{1}{2} \beta_{2}^{i}\left(\omega-\omega_{0}\right)^{2}+\frac{1}{6} \beta_{3}^{i}\left(\omega-\omega_{0}\right)^{3}+\cdots
$$


where $\beta_{0}^{i}, \beta_{1}^{i}, \beta_{2}^{i}, \beta_{3}^{i}$ denote the propagation constant, inverse group velocity (IVG), group velocity dispersion (GVD), and third-order dispersion at the reference frequency $\omega_{0}$ in the mode $i=01,11,02,31$, respectively.

For example, the phase mismatch $\Delta \beta$ for the $\mathrm{BS}$ process between $\mathrm{LP}_{01}$ and $\mathrm{LP}_{11}$ is obtained as:

$$
\begin{aligned}
\Delta \beta & =\beta^{11}\left(\omega_{\mathrm{P}_{2}}\right)+\beta^{01}\left(\omega_{\mathrm{s}}\right)-\beta^{01}\left(\omega_{\mathrm{P}_{1}}\right)-\beta^{11}\left(\omega_{\mathrm{i}_{2}}^{\mathrm{bs}}\right) \\
& \approx\left[\beta_{1}^{01}+\beta_{2}^{01}\left(\frac{\omega_{\mathrm{P}_{1}}+\omega_{\mathrm{s}}}{2}-\omega_{0}\right)-\beta_{1}^{11}-\beta_{2}^{11}\left(\frac{\omega_{\mathrm{P}_{2}}+\omega_{\mathrm{i}_{2}}^{\mathrm{bs}}}{2}-\omega_{0}\right)\right]\left(\omega_{\mathrm{s}}-\omega_{\mathrm{P}_{1}}\right)
\end{aligned}
$$

The phase mismatch for the PC process is identical to that of the BS process given by Eq. (1.4) with just replacing with corresponding idler frequency. Note that high-order dispersion terms are neglected here. Furthermore, the phase mismatch for the BS and PC processes between the other pairs of modes can be defined similarly.

The physical meaning of Eq. (1.4) is that the phase-matching conditions for the intermodal BS and PC processes are satisfied, only if the inverse group velocity evaluated at the average frequency of the two waves in one mode is equal to that evaluated at the average frequency in the other mode. The schematic demonstration of these phase-matched BS processes between $\mathrm{LP}_{01}$ and each higher-order mode is depicted in Fig. 2(a). The four pump wavelengths are selected with the same inverse group velocity in corresponding modes, and the BS idler wavelengths at each higher-order mode will satisfy all the four phase-matching conditions simultaneously (labelled by the horizontal dashed line in Fig. 2(a)) if all the average group velocity dispersion curves have similar curvatures. Moreover, the phase mismatch for the PC processes is doomed to dramatically increase since the corresponding idler wavelength lies oppositely on the dispersion curve with respect to the signal wavelength. Therefore, the phase-matched BS process over a broad signal wavelength range are possible when the chromatic dispersion of all the modes are identical in the investigated range, while the efficiency for the PC process is expected to drop quickly when the signal wavelength is moving away from the pump wavelength in the $\mathrm{LP}_{01}$ mode due to the increasing phase mismatch.

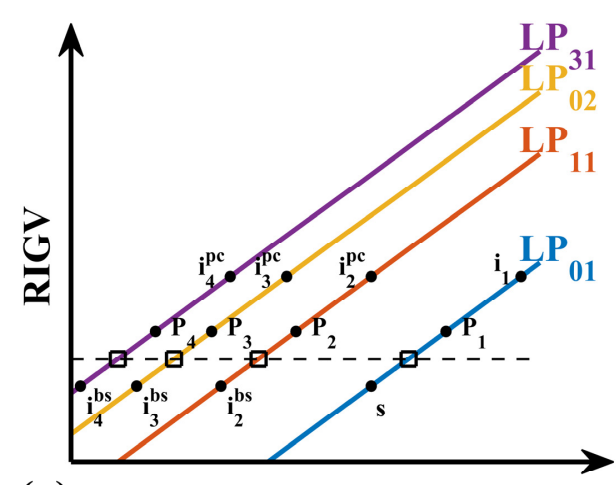

(a) Wavelength

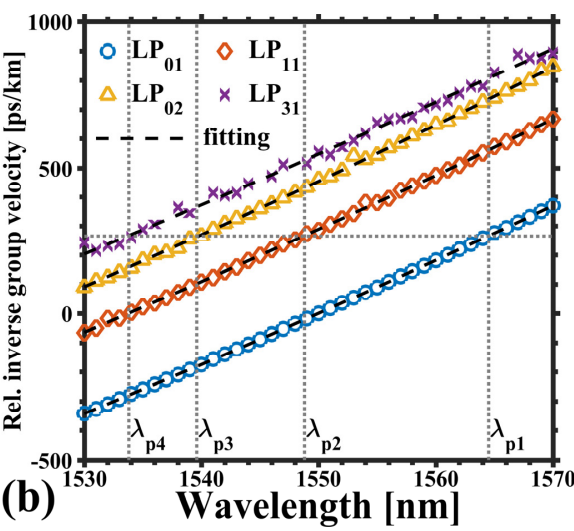

(b)

Wavelength $[\mathbf{n m}]$

Fig. 2. (a) Schematic of the phase-matching conditions of the IFWM between $\mathrm{LP}_{01}$ and higherorder modes. (b) Measurement of the relative inverse group velocity (RIGV) as a function of wavelength for the four modes. The four pump wavelengths $\lambda_{\mathrm{P} 1}-\lambda_{\mathrm{P} 4}$ at the same group velocity in each mode are also shown with dashed lines.

The group velocity dispersion curves of the 6-LP-mode fiber have been measured by the time of flight method, and the values corresponding to the $\mathrm{LP}_{01}, \mathrm{LP}_{11}, \mathrm{LP}_{02}$ and $\mathrm{LP}_{31}$ modes are plotted in Fig. 2(b). The chromatic dispersions (indicated below in the experimental setup 
section) of each mode are obtained by fitting the measured dispersion curves with secondorder polynomials. Besides, the four pump wavelengths $\lambda_{\mathrm{P} 1}-\lambda_{\mathrm{P} 4}$ with the same group velocity are also denoted by dotted lines in Fig. 2(b), which correspond to the wavelength-separation $\Delta \lambda \approx 16 \mathrm{~nm}, 25 \mathrm{~nm}$, and $31 \mathrm{~nm}$ for the $\mathrm{LP}_{11}, \mathrm{LP}_{02}$ and $\mathrm{LP}_{31}$ modes with respect to the $\mathrm{LP}_{01}$ mode.

\subsection{Model}

The IFWM process within each pair of modes can be theoretically described by a set of twomode coupled NLSEs as [15]:

$$
\begin{aligned}
& \frac{\partial E^{(p)}}{\partial z}=\left[i \beta_{0}^{p}-\beta_{1}^{p} \frac{\partial}{\partial t}-\frac{i \beta_{2}^{p}}{2} \frac{\partial^{2}}{\partial t^{2}}+\frac{\beta_{3}^{p}}{6} \frac{\partial^{3}}{\partial t^{3}}+i \gamma\left(f_{p p}\left|E^{(p)}\right|^{2}+2 f_{p q}\left|E^{(q)}\right|^{2}\right)\right] E^{(p)} \\
& \frac{\partial E^{(q)}}{\partial z}=\left[i \beta_{0}^{q}-\beta_{1}^{q} \frac{\partial}{\partial t}-\frac{i \beta_{2}^{q}}{2} \frac{\partial^{2}}{\partial t^{2}}+\frac{\beta_{3}^{q}}{6} \frac{\partial^{3}}{\partial t^{3}}+i \gamma\left(f_{q q}\left|E^{(q)}\right|^{2}+2 f_{p q}\left|E^{(p)}\right|^{2}\right)\right] E^{(q)}
\end{aligned}
$$

where $\gamma=n_{2} \omega / \mathrm{c}$ is the nonlinear Kerr parameter evaluated at the average pump wavelengths, with $\mathrm{c}$ the light velocity in a vacuum and $n_{2}=2.6 \times 10^{-20} \mathrm{~m}^{2} / \mathrm{W}$ the silica Kerr nonlinear refractive index. $\left|E^{(p)}\right|^{2}\left(\left|E^{(q)}\right|^{2}\right)$ is the injected power in the mode $p(q), 1 / f_{p p}$ and $1 / f_{q q}$ are the effective areas of the two interacting modes with $1 / f_{p q}$ the effective coupling area between them.

The interaction of the pump and signal waves in the mode $p$ with the pump and idler waves in the mode $q$ can be resolved by numerical integration of Eqs. (1.5) and (1.6). Alternatively, an analytical solution can be found for the generated idler waves, under the assumptions of undepleted pump waves and small parametric gains for the signal wave. Considering for instance the BS and PC IFWM processes occurring between the $\mathrm{LP}_{01}$ and $\mathrm{LP}_{11}$ modes, the power amplitudes are expressed as:

$$
\begin{aligned}
E^{(01)}(z, t) & =\left\{E_{\mathrm{P} 1}(z) \exp \left[i \beta^{01}\left(\omega_{\mathrm{P}_{1}}\right) z-i \omega_{\mathrm{P}_{1}} t\right]+E_{\mathrm{s}}(z) \exp \left[i \beta^{01}\left(\omega_{\mathrm{s}}\right) z-i \omega_{\mathrm{s}} t\right]\right\} \exp \left(i \omega_{0} t\right)(7) \\
E^{(11)}(z, t) & =\left\{E_{\mathrm{P} 2}(z) \exp \left[i \beta^{11}\left(\omega_{\mathrm{P}_{2}}\right) z-i \omega_{\mathrm{P}_{2}} t\right]+\right. \\
& \left.E_{\mathrm{i}_{2}}(z) \exp \left[i \beta^{11}\left(\omega_{\mathrm{i}_{2}}^{\mathrm{bs}}\right) z-i \omega_{\mathrm{i}_{2}}^{\mathrm{bs}} t\right]+E_{\mathrm{i}_{2}^{\mathrm{pc}}}(z) \exp \left[i \beta^{11}\left(\omega_{\mathrm{i}_{2}}^{\mathrm{pc}}\right) z-i \omega_{\mathrm{i}_{2}}^{\mathrm{pc}} t\right]\right\} \exp \left(i \omega_{0} t\right)
\end{aligned}
$$

Substituting Eqs. (1.7) and (1.8) into Eqs. (1.5) and (1.6) and assuming time-independent electric fields as well as neglecting the nonlinear phase shifts of each wave exerted by $\left|E_{\mathrm{P} 1}\right|^{2}$ and $\left|E_{\mathrm{P} 2}\right|^{2}$ since these terms are small enough compared to linear phase-matching contribution and their effects are mainly to slightly shift the efficiency curves of IFWM with minimum impact on the bandwidth [16], two linear differential equations for the idlers are obtained as:

$$
\begin{aligned}
& \frac{\partial E_{\mathrm{i} 2}(z)}{\partial z}=2 i \gamma f_{01} E_{\mathrm{P} 1}^{*}(z) E_{\mathrm{P} 2}(z) E_{\mathrm{s}}(z) \exp \left(i \Delta \beta^{\mathrm{bs}} z\right) \\
& \frac{\partial E_{\mathrm{p}}^{\mathrm{pc}}(z)}{\partial z}=2 i \gamma f_{01} E_{\mathrm{P} 1}(z) E_{\mathrm{P} 2}(z) E_{\mathrm{s}}^{*}(z) \exp \left(i \Delta \beta^{\mathrm{pc}} z\right)
\end{aligned}
$$

Suppose $E_{\mathrm{P} 1}(z)=E_{\mathrm{P} 1}(0), E_{\mathrm{P} 2}(z)=E_{\mathrm{P} 2}(0)$, and $E_{\mathrm{s}}(z)=E_{\mathrm{s}}(0)$, the idler power for the $\mathrm{BS}$ and $\mathrm{PC}$ processes is: 


$$
\begin{aligned}
& P_{\mathrm{i} \mathrm{bs}}(z)=\left|E_{\mathrm{i}_{2}^{\mathrm{bs}}}(z)\right|^{2}=4 \gamma^{2} f_{01}^{2} z^{2}\left|E_{\mathrm{P} 1}^{*}(0) E_{\mathrm{P} 2}(0) E_{\mathrm{s}}(0)\right|^{2} \sin \mathrm{c}^{2}\left(\Delta \beta^{\mathrm{bs}} z / 2\right) \\
& P_{\mathrm{i}^{\mathrm{pc}}}(z)=\left|E_{\mathrm{i}_{2}^{\mathrm{pc}}}(z)\right|^{2}=4 \gamma^{2} f_{01}^{2} z^{2}\left|E_{\mathrm{P} 1}(0) E_{\mathrm{P} 2}(0) E_{\mathrm{s}}^{*}(0)\right|^{2} \sin \mathrm{c}^{2}\left(\Delta \beta^{\mathrm{pc}} z / 2\right)
\end{aligned}
$$

where $\Delta \beta$ is the phase mismatch defined in Eq. (1.4), $1 / f_{01}$ is the effective coupling area between $\mathrm{LP}_{01}$ and $\mathrm{LP}_{11}$.

It is easily seen from Eqs. (1.11) and (1.12) that the idler power increases quadratically along the fiber at the perfect phase-matching conditions $(\Delta \beta=0)$, until the gain is high enough to deplete the pump powers and energy-exchanges between all the waves happen. For the small gain regime investigated in this work, the conversion efficiency (CE) can be defined as the power ratio of generated idlers with respect to the output signal:

$$
\mathrm{CE}=\frac{P_{\mathrm{i}}(z)}{P_{\mathrm{s}}(z)}=4 \gamma^{2} f_{01}^{2} z^{2}\left|E_{\mathrm{P} 1}\right|^{2}\left|E_{\mathrm{P} 2}\right|^{2} \sin \mathrm{c}^{2}(\Delta \beta z / 2)
$$

So the CE for the BS and PC processes only differs in the phase mismatch $\Delta \beta$ at the same pump powers and fiber length, with $\Delta \beta$ being determined by the wavelength separation of the signal with respect to the pumps. The $\mathrm{CE}$ vs signal wavelength curves for the $\mathrm{BS}$ and $\mathrm{PC}$ processes between $\mathrm{LP}_{01}$ and each higher-order mode, calculated by Eq. (1.13) with input parameters corresponding to ideal experimental conditions, are plotted in Figs. 3(a)-3(c). As mentioned above, we can clearly see that BS process is characterized by a larger bandwidth than PC process.
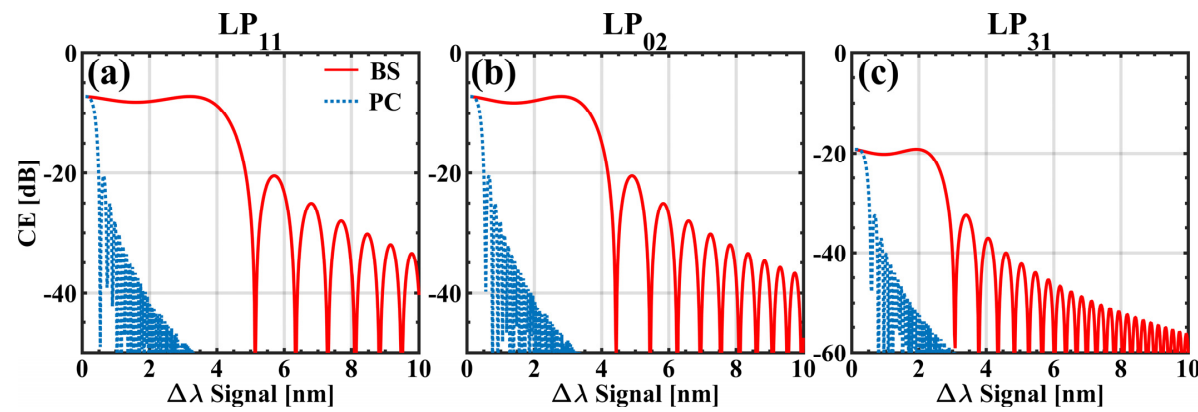

Fig. 3. Theoretical CE vs signal wavelength $\left(\Delta \lambda=\lambda_{\mathrm{P} 1}-\lambda_{\mathrm{s}}\right)$ curves for the BS (red) and PC (blue) processes between $\mathrm{LP}_{01}$ and (a) $\mathrm{LP}_{11}$, (b) $\mathrm{LP}_{02}$, and (c) $\mathrm{LP}_{31}$. The pump wavelength and power are $1564.5 \mathrm{~nm}$ and $28.5 \mathrm{dBm}$ for $\mathrm{LP}_{01}$. The pump wavelength offset $\left(\lambda_{\mathrm{P} 1}-\lambda_{\mathrm{Pn}}\right)$ is $16 \mathrm{~nm}$ for $\mathrm{LP}_{11}$, $24.8 \mathrm{~nm}$ for $\mathrm{LP}_{02}$ as well as $31 \mathrm{~nm}$ for $\mathrm{LP}_{31}$. The pump power in higher-order modes is 20.5 $\mathrm{dBm}$.

It should be also noted that the simultaneous multiple IFWMs conducted in this work are dominated by the respective two-mode BS and PC processes between the fundamental mode and each higher-order mode, and the IFWMs within higher-order modes plays a negligible role considering that the generated idlers are relatively weak compared to the input signal in $\mathrm{LP}_{01}$ (with the conversion efficiency below $-10 \mathrm{~dB}$ in the experiment). Moreover, the genuine injected pump power in $\mathrm{LP}_{01}$ is set to be $8 \mathrm{~dB}$ higher than the other pump powers in higherorder modes during the experiment. Therefore, each higher-order mode interacts with the fundamental mode independently without severe intermodal cross-coupling between them.

Besides, the polarization dependence of the IFWM is not considered in the above scalar NLSEs. The vector nature of the third-order nonlinearity $\chi^{(3)}$ demanding all the participating waves to have parallel polarizations in order to maximize the conversion efficiency. The random birefringence normally present in the $\sim \mathrm{km}$ long fiber will quickly scramble the input state of polarization for all the waves, whose effect can be approximately compensated by a 
reduced nonlinear coefficient $\gamma_{R}=\frac{2}{3} \gamma$ in the Manakov limit [16]. Moreover, since the 6-LPmode fiber used in this work supports four groups of spatial modes, i.e. $\mathrm{LP}_{01}, \mathrm{LP}_{11 \mathrm{a}}+\mathrm{LP}_{11 \mathrm{~b}}$, $\mathrm{LP}_{21 \mathrm{a}}+\mathrm{LP}_{21 \mathrm{~b}}+\mathrm{LP}_{02}$ and $\mathrm{LP}_{31 \mathrm{a}}+\mathrm{LP}_{31 \mathrm{~b}}+\mathrm{LP}_{12 \mathrm{a}}+\mathrm{LP}_{12 \mathrm{~b}}$, the injected power in each higherorder mode will unavoidably spread over all the degenerate modes in the same group if their propagation constants are quite identical. Consequently, the random mode-coupling will also decrease the IFWM efficiency, through the fluctuations of the overlap areas and phasematching conditions between interacting modes. Before ending of this section, the effective coupling areas between the fundamental mode and each higher-order mode are calculated, by approximating the transverse mode profiles by Laguerre-Gaussian functions valid for the graded-index fiber [17]. The values are listed in Table 1 and reveal potentially the weak efficiency of the IFWM between the fundamental mode and the $4^{\text {th }}$ mode group illustrated here by the large effective coupling area of $\mathrm{LP}_{31}$.

Table 1. Effective coupling areas (at $1550 \mathrm{~nm})$ between $L P_{01}$ and higher-order modes in the 6-LP-mode fiber.

\begin{tabular}{|c|c|c|c|c|c|}
\hline $1 / f_{\mathrm{pq}}\left(\mu \mathrm{m}^{2}\right)$ & $\mathrm{LP}_{11}$ & $\mathrm{LP}_{02}$ & $\mathrm{LP}_{21}$ & $\mathrm{LP}_{31}$ & $\mathrm{LP}_{12}$ \\
\hline $\mathrm{LP}_{01}$ & 159 & 159 & 318 & 636 & 212 \\
\hline
\end{tabular}

\section{Experimental setup}

In order to demonstrate the simultaneous threefold modal and wavelength conversion process, we have implemented the experimental setup depicted in Fig. 4. Five tunable external cavity lasers (ECL) in the C-band are used for this experiment. Four different continuous-wave (CW) pumps are first phase-modulated thanks to two phase modulators in such a way to enlarge their spectral linewidth and prevent any backward Brillouin scattering in the fiber undertest. In the case of the fundamental mode, the phase modulator is driven by two amplified voltage-controlled oscillators operating at $96 \mathrm{MHz}$ and $200 \mathrm{MHz}$. For higher-order modes which are pumped with less average power, only one sinusoidal RF signal at $200 \mathrm{MHz}$ is used. Each pump is then amplified by means of single-mode Erbium-doped fiber amplifiers (EFDA) and injected into a 1.8-km long 6-LP-mode fiber. The wavelength and injected power (given at the spatial multiplexer input) in the fundamental mode $\mathrm{LP}_{01}$ are respectively $1564.5 \mathrm{~nm}$ and $33 \mathrm{dBm}$, while the pump power in the three higher-order modes was fixed to $26 \mathrm{dBm}$. The signal to be converted corresponds to a non-return-to-zero (NRZ) 10-Gbit/s $2^{31}$ long data sequence generated from a pulse pattern generator driving an intensity modulator. This optical signal is combined with the $\mathrm{LP}_{01}$ pump wave before amplification thanks to a 90:10 coupler and leads to a power ratio pump/signal above $20 \mathrm{~dB}$. Polarization maintaining fiber pigtails and couplers are used to ensure that pump and signal in the fundamental mode are injected with the same state-of-polarization in the FMF. The input pump and signal waves are then coupled into the FMF fiber by means of a 10-mode spatial multiplexer from Cailabs based on a multi-plane light conversion technology [18]. Polarization controllers are also inserted in each pump optical path in such a way to maximize the conversion efficiency for each mode. 


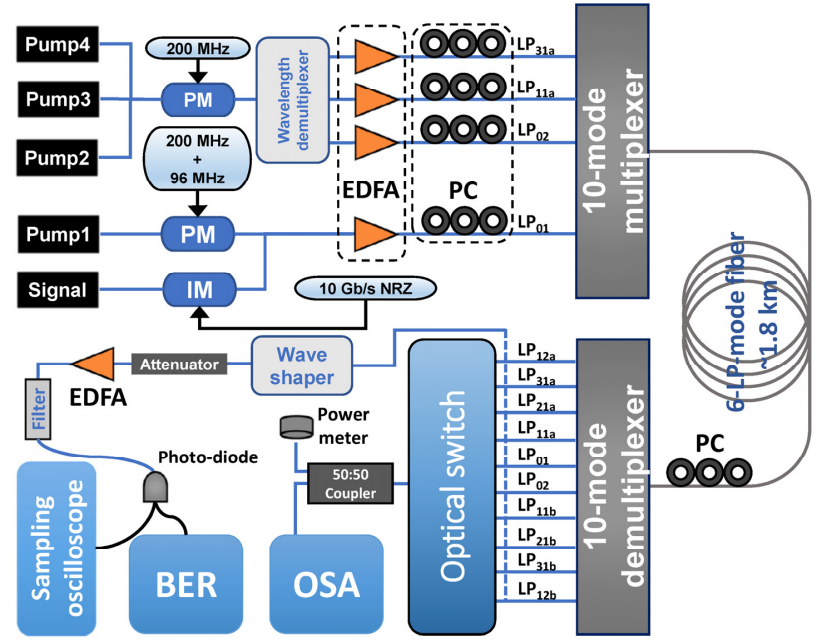

Fig. 4. Overview of the experimental setup. PM phase modulator, IM intensity modulator, PC polarization controller, EDFA Erbium-doped fiber amplifier, OSA optical spectrum analyzer, BER bit-error-rate measurement.

The fiber undertest is a 6-LP-mode graded-index fiber (10 spatial modes including degenerate modes) manufactured by Prysmian group with a core diameter of $22.5 \mu \mathrm{m}$ [19]. The effective areas $\left(\mathrm{A}_{\mathrm{eff}}\right)$ of each supported mode around $1550 \mathrm{~nm}$ are: $75 \mu \mathrm{m}^{2}$ for the fundamental mode $\mathrm{LP}_{01}, 100 \mu \mathrm{m}^{2}$ for $\mathrm{LP}_{11}, 140 \mu \mathrm{m}^{2}$ for $\mathrm{LP}_{21}, 160 \mu \mathrm{m}^{2}$ for $\mathrm{LP}_{02}, 170 \mu \mathrm{m}^{2}$ for $\mathrm{LP}_{31}$ and $170 \mu \mathrm{m}^{2}$ for the $\mathrm{LP}_{12}$. From our measurements reported in Fig. 2(b), the chromatic dispersion is close to $17.5 \mathrm{ps} / \mathrm{nm} / \mathrm{km}$ for the modes $\mathrm{LP}_{12}$ and $\mathrm{LP}_{31}, 18 \mathrm{ps} / \mathrm{nm} / \mathrm{km}$ for $\mathrm{LP}_{01}$ and $\mathrm{LP}_{11}$ and $19 \mathrm{ps} / \mathrm{nm} / \mathrm{km}$ for $\mathrm{LP}_{02}$ and $\mathrm{LP}_{21}$, respectively. The losses for all the modes are below $0.25 \mathrm{~dB} / \mathrm{km}$, and the maximum differential mode group delay (DMGD) between the modes is lower than $550 \mathrm{ps} / \mathrm{km}$. The length of the fiber, $1.8 \mathrm{~km}$, has been arbitrarily chosen in order to achieve a sufficient conversion process. At the fiber output, a 10-mode spatial demultiplexer coupled to a 10-input switch is used to extract and characterize the different modes. Note that due to strong linear mode mixing in the fiber undertest, the output spectra are recorded by adding all the modes from the same spatial group, meaning that output $\mathrm{LP}_{11}$ corresponds in fact to the sum of both demultiplexed degenerated mode $\mathrm{LP}_{11 \mathrm{a}}$ and $\mathrm{LP}_{11 \mathrm{~b}}, \mathrm{LP}_{02}$ to the sum of the three modes $\mathrm{LP}_{02}, \mathrm{LP}_{21 \mathrm{a}}$ and $\mathrm{LP}_{21 \mathrm{~b}}$ while $\mathrm{LP}_{31}$ denotes the sum of $\mathrm{LP}_{31 \mathrm{a}}, \mathrm{LP}_{31 \mathrm{~b}}, \mathrm{LP}_{12 \mathrm{a}}$ and $\mathrm{LP}_{12 \mathrm{~b}}$. Moreover, for temporal characterizations, the injection and demultiplexing conditions (polarization controller and fiber stress before demultiplexing operation) were optimized for each mode undertest. The total losses of the system were measured at an average value of 9.3 $\mathrm{dB}$ for all the modes (slightly better for the fundamental mode) while the average cross-talks between the different groups of modes were found better than $-20 \mathrm{~dB}$. Finally, for temporal characterization, a programmable liquid-crystal-based filter (waveshaper) is used to extract the converted signal in each mode before eye-diagram recording and bit-error-rate (BER) measurements.

\section{Results and discussions}

\subsection{Phase-matching conditions}

We have first experimentally characterized the phase-matching conditions for all the three higher-order modes undertest. To this aim, we have fixed the pump and signal wavelengths to $1564.5 \mathrm{~nm}$ and $1564.0 \mathrm{~nm}$ in the fundamental mode and recorded separately the resulting IFWM spectrum as a function of the second pump wavelength injected into each higher-order mode. Figure 5 summarized the results obtained for each group of modes. 

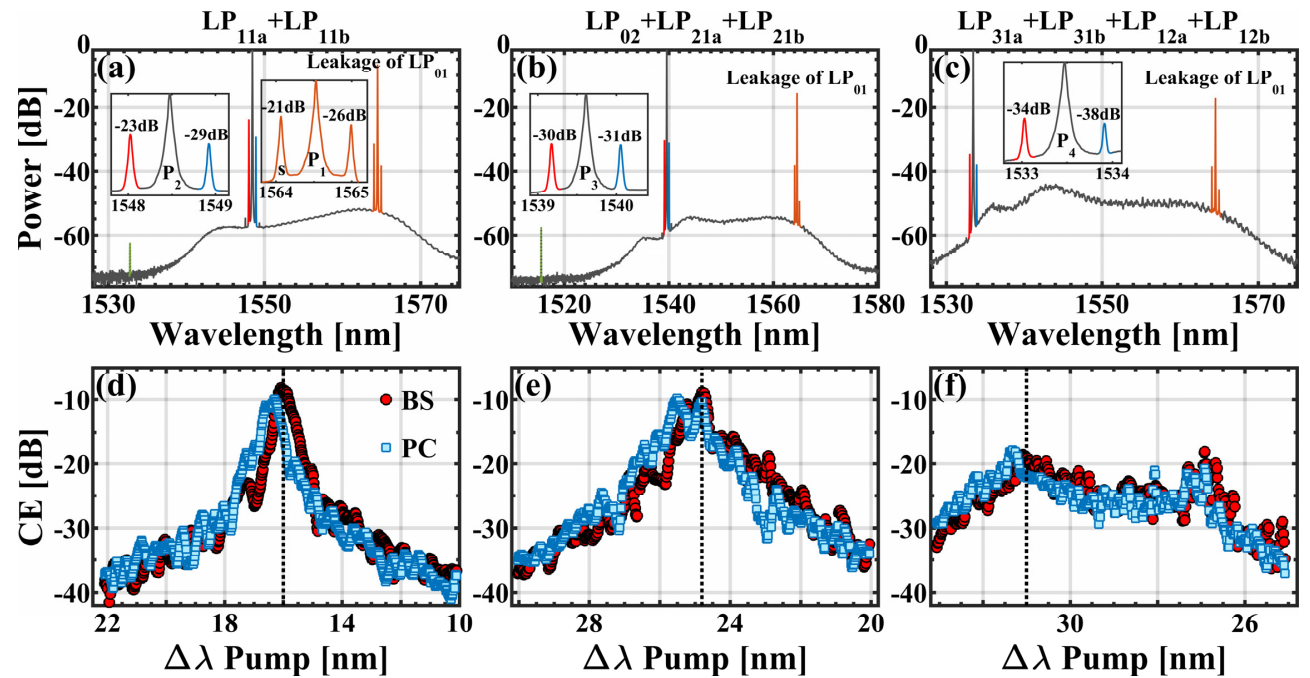

Fig. 5. (a-c) Typical spectrum recorded close to the phase matching condition in each group of modes. (d-f) Conversion efficiency of the Bragg-scattering and phase-conjugation processes observed when scanning the pump wavelengths $\left(\Delta \lambda=\lambda_{\mathrm{P} 1}-\lambda_{\mathrm{Pn}}\right)$ in $\mathrm{LP}_{11}(\mathrm{~d}), \mathrm{LP}_{02}(\mathrm{e})$ and $\mathrm{LP}_{31}(\mathrm{f})$.

The top line shows typical output spectra recorded for the $\mathrm{LP}_{11}$ (Fig. 5(a)), $\mathrm{LP}_{02}$ (Fig. 5(b)) and $\mathrm{LP}_{31}$ group (Fig. 5(c)). For all the higher-order modes, we can clearly observe the generation of two new spectral components surrounding their pump wavelengths, which is originated from the two different IFWM processes referred as Bragg-scattering (BS) and phase-conjugation (PC) introduced in section 2. We can also notice the leakage of the fundamental mode (denoted as orange solid lines in each figure), occurring from the crosstalk mainly introduced by the pair of spatial multiplexer/demultiplexer. Interestingly, a weak and distant wave (plotted as green dashed lines in Figs. 5(a)-5(b)) also appears in the output spectra of higher-order modes, whose frequency corresponds to the mixing of one pump photon in higher-order modes with one of this leakage photon coupled into the fundamental mode and one pump photon in the fundamental mode, i.e. $2 \omega_{\mathrm{P}_{2}}-\omega_{\mathrm{P}_{1}}$. Indeed, we have

verified that this wave is generated by a similar degenerated intermodal BS process exploiting the leakage of pump waves injected into the higher-order modes and remaining within the fundamental mode which they play the role of a 'new' signal wave for the BS IFWM under study.

To fully optimize the phase matching condition, we have reported in the bottom of Fig. 5 the evolution of the conversion efficiency (CE) of the BS and PC processes, as a function of the pump wavelength in higher-order modes. For $\mathrm{LP}_{11}$ modes (Fig. 5(d)), the optimum phase matching condition is obtained for a pump wavelength offset close to $16 \mathrm{~nm}$ compared to the $\mathrm{LP}_{01}$ pump wavelength, in good agreement with the experimental dispersion curves reported in Fig. 2(b). The maximum conversion efficiency for $\mathrm{LP}_{11}$ is close to $-8 \mathrm{~dB}$. Similar results are obtained for the other higher-order modes with an optimum wavelength offset of $24.8 \mathrm{~nm}$ from the $\mathrm{LP}_{01}$ pump wavelength for $\mathrm{LP}_{02}$ and $31 \mathrm{~nm}$ for $\mathrm{LP}_{31}$, respectively, in good agreement with results of Fig. 2(b). We can also notice that the wavelength range of the phase matching process increases with higher-order modes, while the $\mathrm{CE}$ drops to $-18 \mathrm{~dB}$ for $\mathrm{LP}_{31}$. This behavior is attributed to the increasing number of degenerate modes in these groups and their associated random couplings, for which the slight differences in the phase matching condition enlarge the resulting bandwidth at the expense of the conversion efficiency [20,21]. It could be also related to the core-radius fluctuations, which randomly changes the propagation constants of all the modes along the fiber length [22].This issue is quite similar to the case of parametric processes in single mode fibers for which the random fluctuations of the 
dispersion properties along the fiber length, especially the zero-dispersion wavelength, induce a decrease of the conversion efficiency [23]. Note that the optimum pump wavelengths for the BS and PC processes in each higher-order mode are not perfectly overlapped since they correspond to slightly different phase matching conditions. In fact, this small offset is equal to the separation between the pump and signal wavelengths injected into the fundamental mode. This behavior can be easily understood by referring to the different phase matching conditions of the BS and PC processes plotted in Fig. 2(a).

\subsection{Bandwidths of Bragg-scattering and Phase-conjugation}

In order to further characterize the bandwidth and $\mathrm{CE}$ of Bragg-scattering and phaseconjugation processes, we have mapped the IFWM spectrum for fixed pump wavelengths close to the phase matching condition found above, while the signal wavelength injected in $\mathrm{LP}_{01}$ is continuously swept. More precisely, the specific spatial mode, wavelength and power of each pump (measured at the input of the spatial multiplexer) are fixed for $\operatorname{LP}_{01}(1564.5 \mathrm{~nm}$, $33 \mathrm{dBm}), \mathrm{LP}_{11 \mathrm{a}}(1548.8 \mathrm{~nm}, 26 \mathrm{dBm}), \mathrm{LP}_{02}(1539.6 \mathrm{~nm}, 26 \mathrm{dBm})$ and $\mathrm{LP}_{31 \mathrm{a}}(1533.8 \mathrm{~nm}, 26$ $\mathrm{dBm})$, respectively. Note that as already mentioned above, the output spectra are recorded by adding all the modes within the same spatial group. Results are summarized in Fig. 6(a). We can clearly observe the simultaneous generations of BS and PC idler-waves in each group of modes, as well as the wider bandwidth of the BS process compared to that of the PC process (represented by the asymmetric X-shapes in Fig. 6(a)). This phenomenon is well captured by the different sensitivity of phase-mismatch on tuning signal wavelengths for both processes discussed in section 2: the signal and idler in the BS process move in the same direction and thus an identical phase matching condition is maintained, while for the PC process the signal and idler move in opposite directions and a fast deviation from the phase-matched configuration is expected. Therefore, sweeping the signal wavelength will cause rapidly decreasing $\mathrm{CE}$ for the PC process.

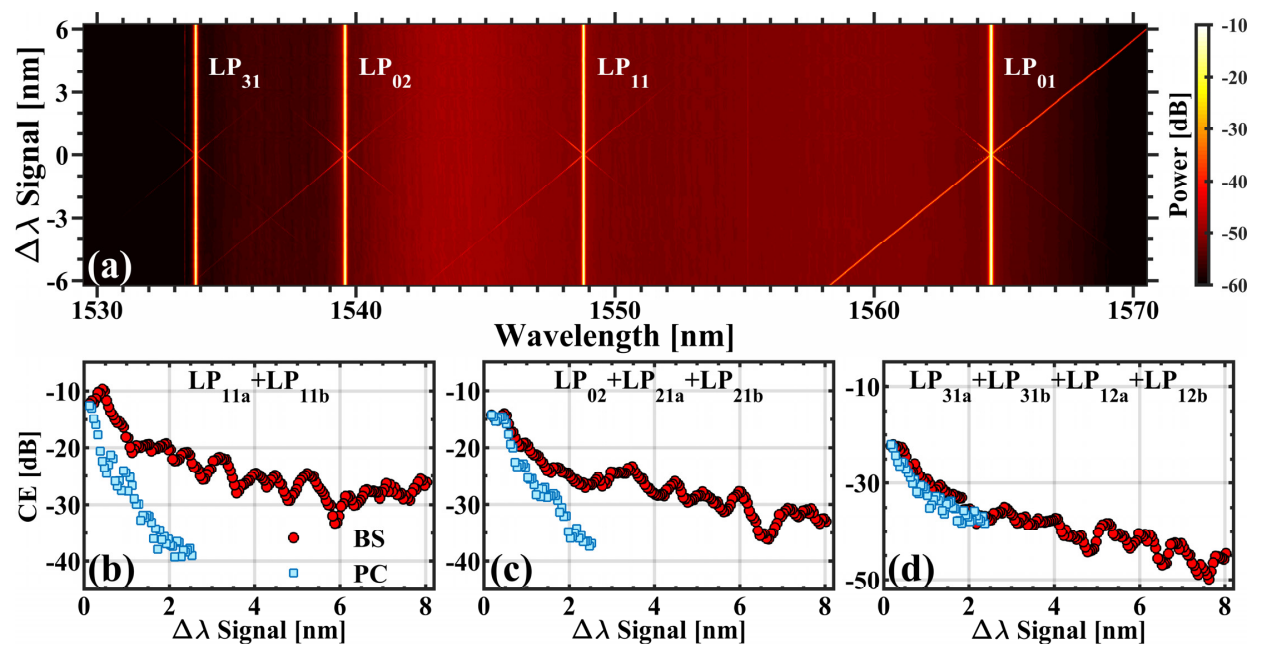

Fig. 6. (a) Experimental measurement of the total output spectrum with respect to the $\mathrm{LP}_{01}$ signal wavelength when pumping the 4 modes simultaneously. The output spectra from each port of the demultiplexer are added to obtain the total spectrum. The spectra are normalized by the pump power measured in the fundamental mode. (b-d) Conversion efficiency of the Braggscattering and phase-conjugation processes measured in each group of modes when scanning the wavelength of the $\mathrm{LP}_{01}$ signal wave. For these data, the output spectra from the same mode-group are summed up to calculate the conversion efficiency.

In order to further illustrate and quantify the different bandwidths of both processes, we have reported for each group of modes in Figs. 6(b)-6(d), the CE with respect to the signal wavelength for the BS (red dots) and PC (blue squares) processes. These curves clearly 
confirm the large difference of bandwidth between both conversion processes, as predicted in Fig. 3. However, the CE for the BS process is also decreased faster, deviating from the above theoretical bandwidth analysis. Indeed, our numerical simulations can be compared only qualitatively with our experimental results since our model does not take into account for all the perturbative effects such as random coupling, random core-radius fluctuations, as well as modal degeneracy, which possibly decrease the IFWM efficiency. This discrepancy can be also attributed to the neglected small differences for the chromatic dispersions of each mode and high-order dispersion terms which destroys the ideal phase matching conditions at large signal-pump wavelength separations. Moreover, the vectorial nature of the propagation, especially the polarization mode dispersion effect is not taken into account in our model, which is known to severely reduce the IFWM gain bandwidth [10,24]. More importantly, the impairment of the $\mathrm{CE}$ with respect to the number of degenerate modes in the same group is observed particularly relevant in Fig. 6(d), where the CE for this group of 4 spatial-modes $(8$ in polarization) is decreased by more than $10 \mathrm{~dB}$ compared to the $\mathrm{LP}_{11}$ group. As already mentioned, for large group of modes, the conversion efficiency is then blurred by the random coupling between adjacent and degenerate modes of the same group [20,21].

\subsection{Simultaneous modal and wavelength conversion of a $10 \mathrm{Gbit} / \mathrm{s}$ NRZ signal}

In order to highlight the potential of multiple parametric conversions in FMF, we have then carried out a proof-of-principle experiment consisting in the simultaneous modal and wavelength conversion of a $10 \mathrm{Gbit} / \mathrm{s}$ telecom signal from the fundamental mode to the $\mathrm{LP}_{11}$, $\mathrm{LP}_{02}$ and $\mathrm{LP}_{31 \mathrm{a}}$ higher-order modes, by means of the intermodal BS process (note that similar results can be achieved in other degenerate modes). The signal consists of a fixed probe signal centered at $1563.6 \mathrm{~nm}\left(0.9 \mathrm{~nm}\right.$ offset from the $\mathrm{LP}_{01}$ pump wavelength) modulated by a 10 Gbit/s NRZ $2^{31}$ data sequence. The wavelength and power of the four pump waves remain identical to the previous CE measurements. At the output of the system, each converted signal is first spatially demultiplexed and then filtered out thanks to the programmable waveshaper, amplified by means of an EDFA and subsequently characterized in the time domain by means of a 12.5-GHz bandwidth photo-detector connected to an electrical sampling oscilloscope.

First-of-all, Fig. 7(a) displays the full optical spectrum recorded at the output of the fiber and shows that converted signals are well present in all the targeted higher-order modes. As already mentioned above and observed in CE measurements, we can notice that the conversion process appears less efficient in the $\mathrm{LP}_{31}$ mode, which can be attributed to its larger overlap area with $\mathrm{LP}_{01}$, the random coupling between the four modes of this spatial group as well as the bad performance of our amplifier in the edge of the C-band. Then, Figs. 7(c)-7(e) display the resulting eye-diagrams recorded for all the demultiplexed and filtered idler-waves as well as in Fig. 7(b) the output $\mathrm{LP}_{01}$ propagating signal. It is important to quote that to achieve the highest signal-to-noise ratio in one particular degenerate mode, the input polarization of its pump wave and output stress on the fiber undertest before the output demultiplexer have to be optimized. Despite this effort, fluctuations over time of the output power as well as quality of the eye diagram have been observed particularly in the highestorder degenerate mode $\left(\mathrm{LP}_{31 \mathrm{a}}\right)$ due to the random evolution of linear mode coupling and polarization drift induced by external perturbations (temperature, vibration...). These fluctuations occur in a time scale of several minutes and can be compensated by readjusting the polarization controllers. For all the converted signals, the corresponding eye-diagrams appear widely opened, but an increasing amount of amplitude jitter can be observed for the highest-order mode. This increasing intensity noise can be attributed to the low CE of BS process in this group of modes as well as the degradation of the signal-to-noise ratio of our EDFA in the lower edge of the C-band, in particular for the $1533.03 \mathrm{~nm}$ signal generated in the $\mathrm{LP}_{31 \mathrm{a}}$ mode. 

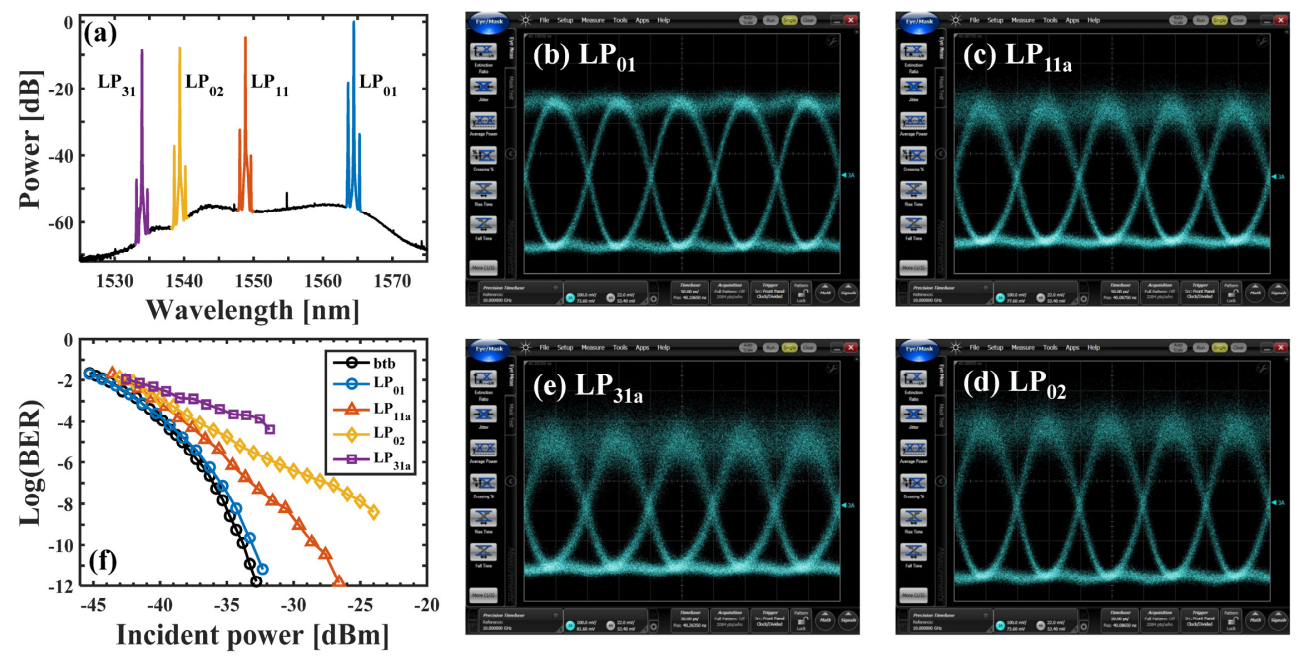

Fig. 7. (a) Optical spectrum of the multiple BS conversion process. (b-e) Eye-diagrams of the output signals in $\mathrm{LP}_{01}, \mathrm{LP}_{11 \mathrm{a}}, \mathrm{LP}_{02}$ and $\mathrm{LP}_{31 \mathrm{a}}$. (f) Bit-error-rate as a function of incoming power on the receiver for all the converted signals as well as back-to-back (btb) measurements.

The performance of this multiple conversion process was then quantitatively characterized by means of bit-error-rate measurements. To this aim, the selected demultiplexed signal was attenuated by means of an automatic attenuator while the BER measurements were performed as a function of the resulting incoming power. Figure 7(f) summarizes these BER measurements in comparison with the back-to-back configuration (black curve). These results show that error-free conversion can be achieved for almost all the generated signals, except for $\mathrm{LP}_{31}$, with an increasing penalty for higher-order modes due to the intensity noise impairments described above.

\section{Conclusion}

In summary, we have experimentally demonstrated a simultaneous threefold wavelength and modal conversion process of a 10-Gbit/s On/Off keying signal in a 1.8-km long graded-index 6-LP-mode fiber. The principle of operation is based on a phase-matched inter-modal fourwave mixing process occurring between the fundamental mode $\mathrm{LP}_{01}$ and 3 higher-order modes, $\mathrm{LP}_{11}, \mathrm{LP}_{02}$ and $\mathrm{LP}_{31}$ of the FMF. The converted signals show well-opened eyediagrams and error-free measurements. The performances of our device are limited by the weak efficiency of IFWM due to large effective areas of the spatial modes in the FMF and the small bandwidth of the involved IFWM process due to modal dispersion, polarization mode dispersion, and chromatic dispersion. Linear coupling, particularly the random coupling between higher order group of modes and degenerate modes is also found to limit the longterm stability of our system. Further improvements could be achieved, in particular the bandwidth of the process, by means of a specific design of the FMF, with lower chromatic dispersion, smaller effective modal areas and the breaking of degeneracy for higher-order modes by means of elliptical core few-mode fibers $[9,24,25]$. One additional perspective could be to generalize this concept to a higher number of spatial modes. In conclusion, this multimode conversion device opens the way to parallel nonlinear signal processing which could find multiple applications in optical communications for the development of agile and flexible SDM networks, sensing, biophotonics or quantum information for the generation of multiple pairs of correlated photons $[22,26]$. 


\section{Funding}

Agence Nationale pour la Recherche, grant (ANR-17-ERC2-0020-01); Conseil Regional de Bourgogne Franche-Comté (project APEX); and FEDER (project Quasimir).

\section{Acknowledgments}

The authors thank B. Kibler, R. Dupiol, P. Bejot and C. Finot for fruitful discussions. We also thank V. Poncet for his precious help on the remote control operation. All these results have benefited from the PICASSO platform in ICB.

\section{References}

1. D. J. Richardson, J. M. Fini, and L. E. Nelson, "Space-division multiplexing in optical fibers," Nat. Photonics 7(5), 354-362 (2013).

2. P. J. Winzer, D. T. Neilson, and A. R. Chraplyvy, "Fiber-optic transmission and networking: the previous 20 and the next 20 years [Invited]," Opt. Express 26(18), 24190-24239 (2018)

3. R. J. Essiambre, G. Kramer, P. J. Winzer, G. J. Foschini, and B. Goebel, "Capacity limits of optical fiber networks," J. Lightwave Technol. 28(4), 662-701 (2010).

4. D. J. Richardson, “Applied physics. Filling the light pipe," Science 330(6002), 327-328 (2010).

5. D. Soma, Y. Wakayama, S. Beppu, S. Sumita, T. Tsuritani, T. Hayashi, T. Nagashima, M. Suzuki, H. Akahashi, K. Igarashi, I. Morita, and M. Suzuki, "10.16 Peta-bit/s dense SDM/WDM transmission over low-DMD 6-mode 19-core fibre across C+L Band," in Proceedings of the European Conference on Optical Communication (ECOC, 2017), paper ThPDPA1.

6. R. J. Essiambre, M. A. Mestre, R. Ryf, A. H. Gnauck, R. W. Tkach, A. R. Chraplyvy, Y. Sun, X. Jiang, and R. Lingle, "Experimental investigation of inter-modal four-wave mixing in few-mode fibers," IEEE Photonics Technol. Lett. 25(6), 539-542 (2013).

7. S. M. M. Friis, I. Begleris, Y. Jung, K. Rottwitt, P. Petropoulos, D. J. Richardson, P. Horak, and F. Parmigiani, "Inter-modal four-wave mixing study in a two-mode fiber," Opt. Express 24(26), 30338-30349 (2016).

8. M. Guasoni, "Generalized modulational instability in multimode fibers: Wideband multimode parametric amplification," Phys. Rev. A 92(3), 033849 (2015).

9. F. Parmigiani, P. Horak, Y. Jung, L. Grüner-Nielsen, T. Geisler, P. Petropoulos, and D. J. Richardson, “Alloptical mode and wavelength converter based on parametric processes in a three-mode fiber," Opt. Express 25(26), 33602-33609 (2017)

10. M. Guasoni, F. Parmigiani, P. Horak, J. Fatome, and D. J. Richardson, "Intermodal four-wave-mixing and parametric amplification in km-long multi-mode fibers," J. Lightwave Technol. 35(24), 5296-5305 (2017).

11. G. Rademacher, R. Ryf, N. K. Fontaine, H. Chen, R. M. Jopson, R. J. Essiambre, B. J. Puttnam, R. S. Luis, Y. Awaji, N. Wada, S. Gross, N. Riesen, M. Withford, Y. Sun, and R. Lingle, "Experimental Investigation of Parametric Mode and Wavelength Conversion in a $4.7 \mathrm{~km}$ Few-Mode Fiber," in 2018 European Conference on Optical Communication (ECOC, 2018), pp. 1-3.

12. J. Hansryd, P. A. Andrekson, M. Westlund, J. Li, and P. O. Hedekvist, "Fiber-based optical parametric amplifiers and their applications," IEEE J. Sel. Top. Quantum Electron. 8(3), 506-520 (2002).

13. R. Slavík, F. Parmigiani, J. Kakande, C. Lundström, M. Sjödin, P. A. Andrekson, R. Weerasuriya, S. Sygletos, A. D. Ellis, L. Grüner-Nielsen, D. Jakobsen, S. Herstrøm, R. Phelan, J. O’Gorman, A. Bogris, D. Syvridis, S. Dasgupta, P. Petropoulos, and D. J. Richardson, "All-optical phase and amplitude regenerator for nextgeneration telecommunications systems," Nat. Photonics 4(10), 690-695 (2010).

14. R. H. Stolen, J. E. Bjorkholm, and A. Ashkin, "Phase-matched-stimulated four-photon mixing in silica-fiber waveguides," Quantum Electron. 11(3), 100-103 (1975).

15. R. Dupiol, A. Bendahmane, K. Krupa, J. Fatome, A. Tonello, M. Fabert, V. Couderc, S. Wabnitz, and G. Millot, "Intermodal modulational instability in graded-index multimode optical fibers," Opt. Lett. 42(17), 3419-3422 (2017).

16. Y. Xiao, R. J. Essiambre, M. Desgroseilliers, A. M. Tulino, R. Ryf, S. Mumtaz, and G. P. Agrawal, "Theory of intermodal four-wave mixing with random linear mode coupling in few-mode fibers," Opt. Express 22(26), 32039-32059 (2014).

17. A. Mafi, "Pulse Propagation in a Short Nonlinear Graded-Index Multimode Optical Fiber," J. Lightwave Technol. 30(17), 2803-2811 (2012).

18. G. Labroille, P. Jian, N. Barré, B. Denolle, and J. Morizur, "Mode Selective 10-Mode Multiplexer based on Multi-Plane Light Conversion," in Optical Fiber Communication Conference (Optical Society of America, 2016), paper Th3E5.

19. P. Sillard, D. Molin, M. Bigot-Astruc, K. de Jongh, and F. Achten, "Micro-Bend-Resistant Low-DMGD 6-LPMode Fiber," in Optical Fiber Communication Conference (Optical Society of America, 2016), paper Th1J5.

20. O. F. Anjum, M. Guasoni, P. Horak, Y. Jung, P. Petropoulos, D. J. Richardson, and F. Parmigiani, "Polarization insensitive four wave mixing based wavelength conversion in few-mode optical fibers," J. Lightwave Technol. 36(17), 3678-3683 (2018). 
Optics EXPRESS

21. M. Esmaeelpour, R. Essiambre, N. K. Fontaine, R. Ryf, J. Toulouse, Y. Sun, and R. Lingle, "Power Fluctuations of Intermodal Four-Wave Mixing in Few-Mode Fibers," J. Lightwave Technol. 35(12), 2429-2435 (2017).

22. J. G. Koefoed, S. M. M. Friis, J. B. Christensen, and K. Rottwitt, "Spectrally pure heralded single photons by spontaneous four-wave mixing in a fiber: reducing impact of dispersion fluctuations," Opt. Express 25(17), 20835-20849 (2017).

23. M. Karlsson, "Four-wave mixing in fibers with randomly varying zero dispersion wavelength," J. Opt. Soc. Am. B 15(8), 2269-2275 (1998).

24. O. F. Anjum, P. Horak, Y. Jung, M. Suzuki, Y. Yamamoto, T. Hasegawa, P. Petropoulos, D. J. Richardson, and F. Parmigiani, "Bandwidth enhancement of inter-modal four wave mixing Bragg scattering by means of dispersion engineering," APL Photonics 4(2), 022902 (2019).

25. M. Guasoni, F. Parmigiani, P. Horak, and D. J. Richardson, "Novel Fiber Design for Wideband Conversion and Amplification in Multimode Fibers," in 2017 European Conference on Optical Communication (ECOC, 2017), pp. 1-3.

26. C. Guo, J. Su, Z. Zhang, L. Cui, and X. Li, "Generation of telecom-band correlated photon pairs in different spatial modes using few-mode fibers," Opt. Lett. 44(2), 235-238 (2019). 